\title{
Case Report: Clostridium neonatale Bacteremia in a Preterm Neonate With Necrotizing Enterocolitis
}

\author{
Nadim Cassir ${ }^{1,2 *}$, Isabelle Grandvuillemin ${ }^{3}$, Manon Boxberger ${ }^{1,2}$, Priscilla Jardot ${ }^{1,2}$, \\ Farid Boubred $^{3,4}$ and Bernard La Scola ${ }^{1,2}$ \\ ${ }^{1}$ Department of Clinical Microbiology, Aix Marseille Université, Institut de Recherche pour le Développement, Assistance \\ Publique des Hôpitaux de Marseille, Microbes, Evolution, Phylogénie et Infection, Marseille, France, ${ }^{2}$ Institut \\ Hospitalo-Universitaire Méditerranée Infection, Marseillle, France, ${ }^{3}$ Department of Neonatology, Hôpital La Conception, \\ Assistance Publique des Hôpitaux de Marseille, AMU, Marseille, France, ${ }^{4}$ Neonatal Unit, C2 VN, Hospital University La \\ Conception, Assistance Publique des Hôpitaux de Marseille, AMU, Marseille, France
}

Necrotizing enterocolitis is a life-threatening acquired gastrointestinal disorder among preterm neonates and is associated with a high mortality rate and long-term neurodevelopmental morbidity. No etiologic agent has been definitively established; nonetheless, the most implicated bacteria include members of the Clostridium genus. We reported here on a case of Clostridium neonatale bacteremia in a preterm neonate with necrotizing enterocolitis, providing more information regarding the potential role of this bacterium in pathogenesis of necrotizing enterocolitis. We emphasized the sporulating form of $C$. neonatale that confers resistance to disinfectants usually applied for the hospital environmental cleaning. Further works are needed to establish the causal relationship between the occurrence of NEC and the isolation of $C$. neonatale, with promising perspectives in terms of diagnostic and therapeutic management.

Keywords: case report, necrotizing enterocolitis, preterm neonate, Clostridium neonatale, gut microbiota

\section{INTRODUCTION}

Necrotizing enterocolitis is a life-threatening acquired gastrointestinal disorder among preterm neonates, especially those of very low birth weight $(<1,500 \mathrm{~g})$ for which the incidence reaches $7 \%$ (1). Necrotizing enterocolitis (NEC) is associated with a high mortality rate (15-30\%) and long-term neurodevelopmental morbidity (1). Although NEC pathophysiology remains unclear, prematurity, enteral feeding strategies, gut bacterial colonization, and inappropriate proinflammatory response are major factors involved in NEC development (1). Gut dysbiosis is considered the cornerstone of NEC pathogenesis. One of the main factors leading to gut dysbiosis is pre- and post-natal antibiotic exposure (1).

There is evidence of the role of specific bacteria in the pathogenesis of NEC. Indeed, outbreaks of NEC have long been described, although the reported causal agents greatly differed (1). Pneumatosis intestinalis is one of the specific radiological signs of NEC that likely represents submucosal gas produced by bacterial fermentation (2). In addition, it has been shown that NEC could not be reproduced in germ-free animals and rarely occurs until at least 8-10 days postpartum in preterm neonates, after strict anaerobic bacterial populations establishment (2).

We report here on a case of Clostridium neonatale bacteremia in a preterm neonate with necrotizing enterocolitis providing more information regarding the potential role of this bacterium in the pathogenesis of NEC. 


\section{CASE}

A $24^{+4}$-week-old gestational age female infant, with a birth weight of $800 \mathrm{~g}$ (95th percentile), was diagnosed with severe necrotizing enterocolitis (NEC) at the day of life (DoL) 21. She was born after cesarean delivery for preterm premature rupture of membranes and after antenatally completing a course of betamethasone. She was immediately admitted to our neonatal intensive care unit (NICU) and required exogenous surfactant combined with nasal continuous positive airway pressure for respiratory distress syndrome. The initial intravenous antibiotics (ampicillin and gentamycin) administered for suspected neonatal infection were stopped on DoL 2. Own breastfeeding was started from DoL1 (colostrum) and was well tolerated. On DoL 21, clinical signs of feeding intolerance suddenly occurred with bilious gastric residuals, abdominal distention, and rectal bleeding. Abdominal X-ray showed pneumatosis intestinalis (Figure 1A). White blood cells count, and C-reactive protein (CRP) respectively peaked at $40 \times 10^{9} / \mathrm{L}$ (normal value range: 4.5 to $11 \times 10^{9} / \mathrm{L}$ ) and $240 \mathrm{mg} / \mathrm{L}$ (normal value $<5 \mathrm{mg} / \mathrm{L}$ ). Enteral feeding was stopped and broad-spectrum antibiotics with vancomycin, cefotaxime, amikacin, and metronidazole were initiated. The yield of blood cultures allowed for the isolation of Clostridium neonatale. More specifically, $24 \mathrm{~h}$ after blood sampling, one anaerobic bottle out of two blood culture sets tested positive. Colony growth was observed on Columbia agar medium (Oxoid, Dardilly, France) supplemented with $5 \%(\mathrm{vol} / \mathrm{vol})$ sheep blood and incubated for $24 \mathrm{~h}$ at $37^{\circ} \mathrm{C}$ in an anaerobic chamber $(80 \% \mathrm{~N} 2,10 \% \mathrm{CO} 2$, and $10 \%$ H2) (AES Chemunex, Bruz, France). Colonies were creamcolored and opaque after $24 \mathrm{~h}$ of growth (Figure 1B). By electronic microscopy using Hitachi SU5000 scanning electron microscope (Hitachi High-Tech Corporation, Tokyo, Japan), colonies were bacilli (Figure 1C). Bacterial cells were Grampositive (not shown). Sporulation was observed after $24 \mathrm{~h}$ under aerobic conditions (Figure 1D). Using matrix-assisted laser desorption ionization-time of flight (MALDI-TOF) mass spectrometry Vitek MS (bioMérieux, France), the organism was identified as Clostridium spp., and this was confirmed as $C$. neonatale through $16 \mathrm{~S}$ rRNA PCR and whole-genome sequencing. In brief, strain Marseille-Q4564 exhibited a 99.66\% $16 \mathrm{~S}$ rRNA sequence similarity with C. neonatale ${ }^{\mathrm{T}}$ (Accession number AF275949.1), the phylogenetically closest bacterium standing in nomenclature. Furthermore, a digital DNA-DNA hybridization revealed a maximum identity similarity of only $91 \%$, and an OrthoANI parameter provided values of 98.89 and 98.95\%, respectively, between Marseille-Q4564 and C. neonatale ${ }^{\mathrm{T}}$ (NZ_PDCJ01000001.1 and NZ_LN890312.1). Taken together, these results confirm the status of this strain belonging to the $C$. neonatale species (Figure 2).

Antimicrobial susceptibility testing was performed. Minimum Inhibitory Concentration (MIC) of tigecycline and vancomycin were determined using Etest (Biomérieux, France), while imipenem, metronidazole, and clindamycin MICs were obtained

Abbreviations: NEC, necrotizing enterocolitis; DoL, days of life; CRP, C-reactive protein. by the disk diffusion method using SIRscan (i2a diagnostics, France). Strain Marseille-Q4564 was susceptible to all the abovementioned antibiotics tested.

While stool culture under anaerobic conditions after a heat shock was negative, specific RT-PCR of C. neonatale tested on stools was positive (3).

Because of a sub-occlusive digestive syndrome with systemic inflammation, surgery was performed 7 days after NEC (DoL28). A severe ischemic jejunum injury was found and was treated with a conservative ileostomy. Culture and RT-PCR specific for C. neonatale tested on the intestine fragment were negative. Cefotaxime and metronidazole were continued for 2 weeks postsurgery. Prolonged parenteral nutrition with minimal enteral feeding was required and preserved adequate growth and a good outcome at 6 months follow-up.

\section{DISCUSSION}

Regarding necrotizing enterocolitis (NEC), several causative microorganisms have been proposed. Nonetheless, the most implicated bacteria include members of the Clostridium genus, especially Clostridium butyricum, C. perfringens, C. paraputrificum, and C. neonatale. Indeed, C. butyricum has frequently been recovered from biological samples of premature neonates suffering from NEC (4). Additionally, in quail and chicken animal models of NEC, C. butyricum, C. perfringens, and C. paraputrificum were shown to be responsible for NEC-like lesions (5). C. neonatale and C. butyricum were reported to be significantly overrepresented among colonic mucosal samples from premature piglets with NEC (6). In 2002, an outbreak of NEC occurred in a Canadian neonatal intensive care unit (7). Blood cultures from three out of six premature neonates grew the same strain further described as C. neonatale, belonging to cluster I of the Clostridium genus sensu stricto (8). Another research team that performed microbiota analysis in 16 cases and 78 controls showed an association between the isolation of Clostridium neonatale from the stools of preterm neonates and the occurrence of NEC (9). A specific rpoB-based quantitative real-time PCR has been recently designed and developed to detect $C$. neonatale directly from the stool specimens of patients. In a case-control study, $C$. neonatale was significantly more prevalent in stools from preterm neonates with NEC than in controls (respectively 30/88 (34\%) vs. 9/71 (13\%); $p=.003$ ) (3). By whole-genome sequencing, genes encoding the secretion of bacterial toxins, especially hemolysins and $C$. difficile toxins $\mathrm{A} / \mathrm{B}(\mathrm{Tcd} \mathrm{A} / \mathrm{B})$ had been previously found (10). These latter are involved in the pathogenic mechanism of $C$. difficile colitis, which is the consequence of TcdA (enterotoxin) and TcdB (cytotoxin) production inducing colonic tissue damage (1).

The preterm neonate acquires its microbiota within the confines of the NICU where colonization is influenced by iatrogenic manipulations including the hospital environment. Recently, an outbreak of NEC was epidemiologically linked to sporulating C. butyricum contamination within the hospital (11). Knowing that spore-forming bacteria are highly resistant to usual hospital disinfectants, sporicidal agents should be used 


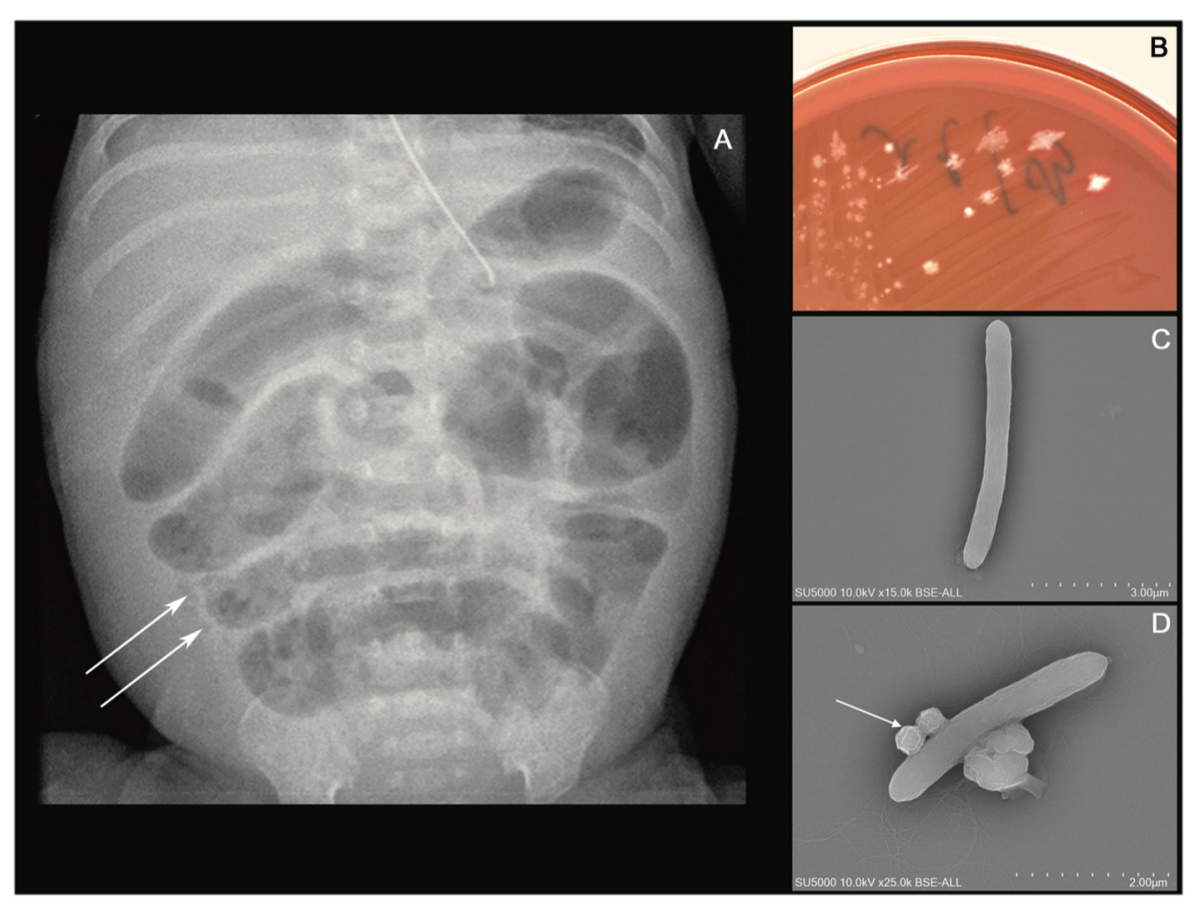

FIGURE 1 | (A) Abdominal X-ray showing intramural bowel gas also known as pneumatosis intestinalis, (B) culture growth of cream-colored colonies on COS after $24 \mathrm{~h}$ of incubation at $37^{\circ} \mathrm{C}$ in the anaerobic chamber, (C) Clostridium neonatale rod-shaped cells using Hitachi SU5000 scanning electron microscope. Scale bar and acquisition settings are shown on the original micrograph, and (D) sporulating C. neonatale cell.

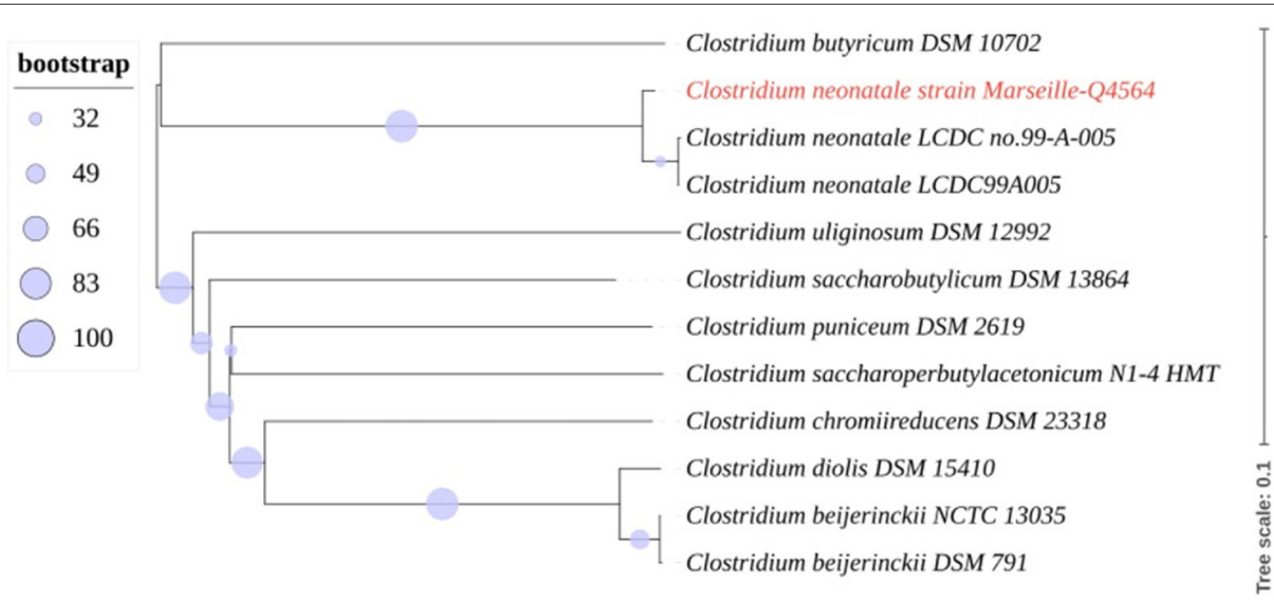

FIGURE 2 | Whole-genome (nucleotides) based phylogenetic tree highlighting the position of C. neonatale strain Marseille-Q4564 relative to other closely related bacterial taxa.

for environmental cleaning in case of sporulating C. neonatale infection. Therefore, we hypothesized that enteric isolation precautions like those applied for patients with $C$. difficile colitis may be effective in controlling NEC outbreaks.

Perinatal exposure to antibiotics has been identified as a risk factor for NEC, in particular when administered more than 5 days (12). The postnatal antibiotic exposure, in this case, may have increased the risk of NEC. Moreover, in this case, the use of large spectrum antibiotics during the episode of NEC may have participated in the secondary worsening 7 days after the initial diagnosis of NEC, by promoting gut dysbiosis. The length of treatment and choice of antimicrobial agents for presumed and proven episodes of NEC vary among centers due to a lack of supportive evidence and guidelines. The study of Cantey et al. showed recently that the implementation of antibiotic stewardship programs including appropriate dosages 
and narrow-spectrum regimens was effective in reducing unnecessary antibiotic use in NICUs (13). In addition, probiotics have been extensively studied to mitigate gut dysbiosis in preterm neonates and prevent the occurrence of NEC (14). A low to moderate level of certainty about the effects of probiotic supplementation on the risk of NEC has been reported. To date, product safety and quality remain of concern especially in a vulnerable population such as preterm neonates. Therefore, probiotics are not routinely used in our NICU. In this case, we cannot rule out the possibility that the administration of a combination of different strains of probiotics may have prevented the occurrence of NEC. Further works are needed to address this issue.

Cultivation of strictly anaerobic species is challenging. Only a few studies analyzing the gut bacterial profile associated with NEC have used strategies to optimize the growth of strictly anaerobic bacteria such as heat shock for sporulating anaerobes, direct inoculation in anaerobic culture bottles, selective culture media, and the use of an anaerobic chamber (14). However, it is too early to state that routine microbiological exams in preterm neonates with NEC should include bacterial culture under anaerobic conditions and RT-PCR testing specific for C. neonatale, C. butyricum, and C. perfringens on a stool, blood, and surgical samples. This case of Clostridium neonatale bacteremia in a preterm neonate with necrotizing enterocolitis provides more information regarding the potential role of this bacterium in the pathogenesis of necrotizing enterocolitis. We hypothesize that the pathophysiological mechanism of $C$. neonatale may be like that of toxigenic $C$. difficile in adults with pseudomembranous colitis. Further works are needed to establish the causal relationship between the occurrence of NEC and the isolation of C. neonatale (15), with promising perspectives in terms of diagnostic and therapeutic management.

\section{DATA AVAILABILITY STATEMENT}

The datasets presented in this study can be found in online repositories. The names of the repository/repositories and

\section{REFERENCES}

1. Cassir N, Simeoni U, La Scola B. Gut microbiota and the pathogenesis of necrotizing enterocolitis in preterm neonates. Future Microbiol. (2016) 11:273-92. doi: $10.2217 / \mathrm{fmb} .15 .136$

2. Neu J, Walker WA. Necrotizing enterocolitis. N Engl J Med. (2011) 364:25564. doi: 10.1056/NEJMra1005408

3. Hosny M, Baptiste E, Levasseur A, La Scola B. Molecular epidemiology of Clostridium neonatale and its relationship with the occurrence of necrotizing enterocolitis in preterm neonates. New Microbes New Infect. (2019) 32:100612. doi: 10.1016/j.nmni.2019.100612

4. Cassir N, Benamar S, Khalil JB, Croce O, Saint-Faust M, Jacquot A, et al. Clostridium butyricum strains and dysbiosis linked to necrotizing enterocolitis in preterm neonates. Clin Infect Dis. (2015) 61:110715. doi: $10.1093 / \mathrm{cid} / \mathrm{civ} 468$

5. Waligora-Dupriet A-J, Dugay A, Auzeil N, Huerre M, Butel M-J. Evidence for clostridial implication in necrotizing enterocolitis through bacterial fermentation in a gnotobiotic quail model. Pediatr Res. (2005) 58:62935. doi: 10.1203/01.PDR.0000180538.13142.84 accession number(s) can be found below: https://www.ncbi.nlm. nih.gov/genbank/, NZ_PDCJ01000001.1.

\section{ETHICS STATEMENT}

Ethical review and approval was not required for the study on human participants in accordance with the local legislation and institutional requirements. Written informed consent to participate in this study was provided by the participant's legal guardian/next of kin.

\section{AUTHOR CONTRIBUTIONS}

NC: conceptualized and designed the study, drafted the initial manuscript, and reviewed and revised the manuscript. IG, MB, and PJ: designed the data collection instruments, collected data, carried out the initial analyses, reviewed, and revised the manuscript. BL and FB: conceptualized and designed the study, supervised data collection, performed analysis and interpretation of data, and critically reviewed the manuscript for important intellectual content. All authors approved the final manuscript as submitted and agree to be accountable for all aspects of the work.

\section{FUNDING}

This research was supported by the French Government under the Investissements d'avenir (investments for the future) program managed by the Agence Nationale de la Recherche grant number [10-IAHU-03].

\section{ACKNOWLEDGMENTS}

We acknowledge Lina Barrassi, Anthony Fontanini, and Ludivine Brechard for their technical assistance.
6. Azcarate-Peril MA, Foster DM, Cadenas MB, Stone MR, Jacobi SK, Stauffer $\mathrm{SH}$, et al. Acute necrotizing enterocolitis of preterm piglets is characterized by dysbiosis of ileal mucosa-associated bacteria. Gut Microbes. (2011) 2:23443. doi: $10.4161 /$ gmic.2.4.16332

7. Alfa MJ, Robson D, Davi M, Bernard K, Van Caeseele P, Harding GKM. An outbreak of necrotizing enterocolitis associated with a novel clostridium species in a neonatal intensive care unit. Clin Infect Dis. (2002) 35:S101105. doi: $10.1086 / 341929$

8. Bouvet P, Ferraris L, Dauphin B, Popoff M-R, Butel MJ. Aires J. 16S rRNA gene sequencing, multilocus sequence analysis, and mass spectrometry identification of the proposed new species "Clostridium neonatale." J Clin Microbiol. (2014) 52:4129-36. doi: 10.1128/JCM.00477-14

9. Rozé JC, Ancel PY, Lepage P, Martin-Marchand L, Al Nabhani Z, Delannoy $\mathrm{J}$, et al. Nutritional strategies and gut microbiota composition as risk factors for necrotizing enterocolitis in very-preterm infants. Am J Clin Nutr. (2017) 106:821-30. doi: 10.3945/ajcn.117.152967

10. Benamar S, Cassir N, La Scola B. Genome sequence of a clostridium neonatale strain isolated in a Canadian neonatal intensive care unit. Genome Announc. (2016) 4:e01431-15. doi: 10.1128/genomeA.01431-15 
11. Dong Y, Li Y, Zhang D, Nguyen S, Maheshwari N, Hu Y, et al. Epidemiological and genetic characterization of Clostridium butyricum cultured from neonatal cases of necrotizing enterocolitis in China. Infect Control Hosp Epidemiol. (2020) 41:900-7. doi: 10.1017/ice.2019.289

12. Cotten CM, Taylor S, Stoll B, Goldberg RN, Hansen NI, Sánchez PJ, et al. Prolonged duration of initial empirical antibiotic treatment is associated with increased rates of necrotizing enterocolitis and death for extremely low birth weight infants. Pediatrics. (2009) 123:58-66. doi: 10.1542/peds.2007-3423

13. Cantey JB, Wozniak PS, Pruszynski JE, Sánchez PJ. Reducing unnecessary antibiotic use in the neonatal intensive care unit (SCOUT): a prospective interrupted time-series study. Lancet Infect Dis. (2016) 16:1178-84. doi: 10.1016/S1473-3099(16)30205-5

14. Murphy K, Ross RP, Ryan CA, Dempsey EM, Stanton C. Probiotics, prebiotics, and synbiotics for the prevention of necrotizing enterocolitis. Front Nutr. (2021) 8:667188. doi: 10.3389/fnut.2021.667188

15. Cassir N, Benamar S, La Scola B. Clostridium butyricum: from beneficial to a new emerging pathogen. Clin Microbiol Infect. (2016) 22:3745. doi: 10.1016/j.cmi.2015.10.014
Conflict of Interest: The authors declare that the research was conducted in the absence of any commercial or financial relationships that could be construed as a potential conflict of interest.

Publisher's Note: All claims expressed in this article are solely those of the authors and do not necessarily represent those of their affiliated organizations, or those of the publisher, the editors and the reviewers. Any product that may be evaluated in this article, or claim that may be made by its manufacturer, is not guaranteed or endorsed by the publisher.

Copyright (C) 2021 Cassir, Grandvuillemin, Boxberger, Jardot, Boubred and La Scola. This is an open-access article distributed under the terms of the Creative Commons Attribution License (CC BY). The use, distribution or reproduction in other forums is permitted, provided the original author(s) and the copyright owner(s) are credited and that the original publication in this journal is cited, in accordance with accepted academic practice. No use, distribution or reproduction is permitted which does not comply with these terms. 